\title{
DIE ENTWICKLUNG DES MODERNEN KONSTITUTIONALISMUS IN LATEINAMERIKA
}

\section{THE EVOLUTION OF MODERN CONSTITUTIONALISM IN LATIN AMERICA}

\author{
Horst Dippel \\ Universität Kassel
}

\begin{abstract}
Resumen: Ausgehend von der These, dass sich 1776 in Nordamerika das Modell des modernen Konstitutionalismus mit zehn Verfassungsprinzipien herausbildete, das mit der Französischen Revolution auf Europa übergriff und hier im Laufe der Zeit übernommen wurde, soll gezeigt werden, dass sich diese Entwicklung ebenso in Lateinamerika mit dem Beginn der Verfassungsgebung nachzeichnen lässt. Ungeachtet vieler herausragender Beispiele im 19. Jahrhundert blieben universelle Prinzipien, der Vorrang der Verfassung, begrenzte Regierung und richterliche Unabhängigkeit bis in die 1970er Jahre am stärksten umkämpft. Kulturelle Prägungen und ethnische Bedingungen führen heute zu weiteren Angleichungen oder Sonderformen.
\end{abstract}

\begin{abstract}
Originating in North America in 1776, modern constitutionalism with its ten core principles was taken up in the French Revolution and spread across Europe during the subsequent centuries. The same became true for Latin America from the early $19^{\text {th }}$ century on with an amazing number of $19^{\text {th }}$ century constitutions documenting this process. However, until the 1970s universal principles, constitutional supremacy, limited government, and judicial independence were the principles most heavily embattled due to major cultural differences and legal education. In recent decades, modern constitutionalism made further progress while at the same time due to the ethnic make-up several countries tried their own way.
\end{abstract}

Palabras clave: Moderner Konstitutionalismus, lateinamerikanische Verfassungen, Unabhängigkeit der Justiz, Vorrang der Verfassung, Präsidentialismus, soziokulturelle Prägungen

Keywords: Modern constitutionalism, Latin American constitutions, judicial independence, constitutional supremacy, presidentialism, social and cultural impact

In seiner weit verbreiteten Untersuchung Modern Constitutions since 1787 von 1939 nannte John A. Hawgood als Entschuldigung, warum er in ihr die lateinamerikanischen Verfassungen nicht beachtet habe, „that the very people for whom these constitutions were designed have themselves provided him with a precedent in not paying overmuch attention to them" ${ }^{1}{ }^{1}$ Ganz so weit wollte Hans Fenske 2001 nicht gehen und widmete immerhin 30 (von 570) Seiten dem schon

1 John A. Hawgood, Modern Constitutions since 1787, London: Macmillan, 1939, vi. 
bezeichnend formulierten Kapitel „Lateinamerika und der Verfassungsstaat“. Sein Fazit unterschied sich jedoch kaum von dem Hawgoods: Es gäbe keine originellen Beiträge, mangele es Lateinamerika doch an jenen, die für „ein geordnetes Verfassungsleben unter rechtlichen Normen“ einträten. ${ }^{2}$ Die Beispiele ließen sich, zumal aus dem nordatlantischen Raum, fortsetzen. Dabei ist unbestritten, dass die Verfassungsentwicklung in Lateinamerika anders verlaufen ist als in den Vereinigten Staaten von Amerika und als in zahlreichen Staaten Europas. Doch berechtigen eine mitunter gehäufte Verfassungsinstabilität zu derart pauschalen Urteilen, oder sind diese nicht eher Ausdruck der Ignoranz ihrer Verfasser? Nicht allein die Tatsache, dass die mexikanische Bundesverfassung und in ihrer Folge zahlreiche Einzelstaatsverfassungen des Landes inzwischen ein hundert Jahre alt sind und damit älter sind als die meisten europäischen Verfassungen und etliche Einzelstaatsverfassungen der USA, spricht eine andere Sprache. Ist der moderne Konstitutionalismus also tatsächlich in Lateinamerika grundlegend anders verlaufen als in anderen Teilen der Welt?

Langlebigkeit ist kein Verfassungsprinzip, sondern allein das Ergebnis einer ausgewogenen Verfassung plus eines ihr entsprechenden politisch-sozialen Umfeldes und der Fähigkeit, unvorhersehbare politische, militärische oder ökonomische Krisen unter Wahrung ihres Grundcharakters lösen zu können. Letzteres setzt einen Konsens über die zentralen Inhalte eben dieses Grundcharakters voraus - es sei denn, man achtet die Verfassung wie im Deutschen Reich von 1871 derart gering, dass es nicht nötig erscheint, einer gewandelten politischen Praxis und ihrer Institutionen durch eine entsprechende Änderung der Verfassung eine verfassungsrechtliche Legitimation zu verschaffen. Der moderne Konstitutionalismus, wie er sich in der amerikanischen und Französischen Revolution im ausgehenden 18. Jahrhundert herausgebildet hatte, verkörperte diesen Konsens, dem die auf beiden Seiten des Nordatlantiks gemeinsam vertretene Überzeugung zugrunde lag, dass es die primäre Aufgabe einer zu konzipierenden Verfassung sei, die Rechte und Freiheiten ihrer Bürger dauerhaft zu sichern. Um dieser Aufgabe gerecht zu werden, bedürfe es zunächst einer Erklärung der Rechte, in der die Prinzipien, auf denen die nachfolgende Verfassung zu gründen sei, festzuschreiben seien.

Die Virginia Declaration of Rights vom 12. Juni 1776 ist das erste Verfassungsdokument, das diese Auffassungen zum Ausdruck bringt. Es ist das Gründungsdokument des modernen Konstitutionalismus, aus dem sich jene zehn Prinzipien herauslesen lassen, die seither den modernen Konstitutionalismus prägen: Volkssouveränität (Art. 2), Menschenrechte (Art. 1), universelle Prinzipien (Art. 1, 15), begrenzte Regierungsgewalt (Art. 3), die Verfassung als oberstes Gesetz (Präambel), repräsentatives Regierungssystem (Art. 6), Gewaltentrennung (Art. 5), Verantwortlichkeit der Regierenden (Art. 2), Unabhängigkeit der Justiz (Art. 8) und die Abänderbarkeit der Verfassung unter Mitwirkung des Volkes (Art. 3). ${ }^{3}$ In wachsender Zahl tauchten diese Prinzipien in

2 Hans Fenske, Der moderne Verfassungsstaat. Eine vergleichende Geschichte von der Entstehung bis zum 20. Jahrhundert, Paderborn usw.: Ferdinand Schöningh, 2001, 470.

3 Vgl. ausführlich dazu Horst Dippel, „Constitucionalismo moderno. Introducción a una Historia que Necesita ser Escrita”, in: Historia Constitucional, 6 (2005), 181-199; Horst Dippel, "El surgimiento del constitucionalismo moderno y las constituciones latinoamericanas tempranas", in: Revista Pensamiento Juridico, 23 (2008), 13-32. In dem funktionalen Verständnis von Verfassung finden sich bei Dieter Grimm, Die Zukunft der Verfassung II: Auswirkungen von 
den nachfolgenden amerikanischen Rechteerklärungen und Verfassungen ebenso auf wie in den französischen Revolutionsverfassungen ab 1789.

Wie kaum anders zu erwarten, beendete Napoleon diesen revolutionären Verfassungstypus des modernen Konstitutionalismus, und auch die nachfolgende Restauration in Frankreich und Europa mit ihrer Abwehr der Ideen von 1789 wollte von diesen Verfassungsprinzipien nichts wissen. Dennoch ließ sich der moderne Konstitutionalismus in Europa in der ersten Hälfte des 19. Jahrhunderts nicht vollends unterdrücken, und die erste Verfassung, in der die Volkssouveränität, das repräsentative Regierungssystem, Gewaltentrennung, begrenzte Regierungsgewalt, die Verantwortlichkeit der Regierenden und die Abänderbarkeit der Verfassung unter indirekter Mitwirkung des Volkes und damit die Mehrheit der zehn Prinzipien wieder auftauchten, war die Verfassung von Cadiz von 1812. Weitere Stationen des modernen Konstitutionalismus in Europa waren dann unter anderem die belgische Verfassung von 1831 und zumal die Verfassungsbestrebungen im Zuge der Revolutionen von 1848, allen voran der Verfassungsentwurf von Kremsier vom 4. März 1849 und die Verfassung der Römischen Republik vom 1. Juli 1849, in denen sich alle zehn Verfassungsprinzipien wiederfanden. ${ }^{4}$ Doch auch für die zweite Hälfte des 19. und das gesamte 20. Jahrhundert blieb ihre Geschichte in Europa wechselhaft, und dramatischen, ja katastrophalen Rückschritten standen beispielgebende Erfolge gegenüber.

Alle diese Entwicklungen waren nicht spurlos an Lateinamerika vorübergegangen. Die nordamerikanischen und französischen Revolutionsverfassungen waren aufmerksam registriert worden. Es hatte das eingesetzt, was man heute in der Verfassungsgeschichtsschreibung die „migration of constitutional ideas“ nennt. 5 Die Verfassung von Cadiz hatte darüber hinaus in weiten Teilen Hispanoamerikas ganz unmittelbare Bedeutung erlangt. ${ }^{6}$ Welche Inhalte notwendig waren, um eine ausgewogene Verfassung zu schaffen, erschien zunehmend klarer, und je länger das nordamerikanische Beispiel währte, umso deutlicher wurde erkennbar, dass es funktionierte.

Dennoch war Lateinamerika anders, und einfache Kopien schieden von vorneherein aus, wie schon die französische Verfassungsgeschichte ungeachtet gleicher Wurzeln im modernen Konstitutionalismus alles andere als eine Kopie der nordamerikanischen, sondern mitunter eher das Gegenteil davon ist. Die englischen Siedler in den nordamerikanischen Kolonien - die indianische

Europäisierung und Globalisierung, Berlin: Suhrkamp, 2012, bes. S. 39-40, 324-325, von diesen zehn Prinzipien die Volkssouveränität und der Vorrang der Verfassung wieder.

4 Vgl. dazu Horst Dippel, "Die Bedeutung der Verfassung der Römischen Republik in der Geschichte des modernen Konstitutionalismus", in: Giornale di Storia costituzionale, VII,1 (2004), 85-90.

5 Vgl. The Migration of Constitutional Ideas, hrg. v. Sujit Choudry, Cambridge: University Press, 2006. Vgl. dazu auch, bezogen auf Spanien und Lateinamerika, die Idee der "Atlántida constitucional“ von José M. Portillo Valdés, „La Atlántida constitucional. La dimensión hispana de las revoluciones de independencia“, in: México: Un siglo de historia constitucional (1808-1917). Estudios y perspectivas, hrg. v. Cecilia Noriega und Alicia Salmerón, México: Instituto Mora, 2009, 3-14.

6 Vgl. Roberto Breña, "La constitución de Cádiz: alcances y límites en Nueva España”, in: México: Un siglo de historia constitucional (1808-1917), hrg. v. Noriega und Salmerón, 15-29. 
Urbevölkerung war ausgerottet oder vertrieben oder, wenn es sie in Resten noch gab, marginalisiert und politisch-sozial ausgeschlossen; die Schwarzen bis auf eine relativ kleine Zahl waren Sklaven - hatten vor 1776 zum Teil jahrzehntelang für ihre Rechte und Freiheiten gekämpft. Politische Unabhängigkeit von Großbritannien war erst in letzter Minute als zusätzliches Ziel aufgetaucht und hatte mehr gespalten denn geeinigt. In Lateinamerika hingegen - und die Gründe hierfür lagen auf beiden Seiten des Atlantiks - ging es, folgt man den Texten, um Unabhängigkeit, Souveränität, Nation. ${ }^{7}$ Damit waren andere politische Prioritäten gesetzt, die ungeachtet ihrer im allgemeinen vergleichsweise geringen politischen Halbwertszeit mit den modernen Verfassungen in Einklang zu bringen waren, obgleich keine von diesen in besonderer Weise auf Lateinamerika und seine vielfältigen Anforderungen zugeschnitten erschien, selbst wenn wir uns allein auf Hispanoamerika beschränken und Haiti mit seinen besonderen Bedingungen und Erfahrungen hier ebenso unberücksichtigt lassen wie zunächst Brasilien. Nicht nur gab letzteres 1826 umgekehrt Europa, nämlich Portugal, eine Verfassung, sondern dokumentierte damit und gemeinsam mit seiner eigenen Verfassung von 1824 auch noch einen weiteren Einfluss im Rahmen der „migration of constitutional ideas“, nämlich den der englischen Verfassung als antirevolutionäres Projekt. 8

Dennoch wirkten die Ideen und Prinzipien des modernen Konstitutionalismus, selbst wenn die Übersetzung der Déclaration des droits de l'homme et $d u$ citoyen von 1789 im Jahr 1793 durch den Revolutionär und nachmaligen Präsidenten von Cundinamarca Antonio Nariño noch eher eine private, wenn auch weit reichende Initiative war. ${ }^{9}$ So war in der Acta de la Confederación de las Provincias Unidas de la Nueva Granada von 1811, abgesehen von dem Bekenntnis zum repräsentativen Regierungssystem, von den Prinzipien des modernen Konstitutionalismus noch nicht viel zu lesen, woran selbst die Reformen von 1814/15 nichts Grundlegendes änderten. ${ }^{10}$

7 Beispielhaft für die Diskussion über den Zusammenhang zwischen Souveränität und Nation, vgl. Manuel Chust, „La constitución de 1812: Una revolución constitucional bihemisférica“, in: El laboratorio constitucional iberoamericano: 1807/1808-1830, hrg. v. Antonio Annino und Marcela Ternavasio, Madrid: AHILA Iberoamericana, 2012, 93-114.

8 Vgl. dazu Horst Dippel, „A Nineteenth-Century ,Truman Doctrine' avant la lettre? Constitutional Liberty Abroad and the Parliamentary Debate about British Foreign Policy from Castlereagh to Palmerston", in: Constitution, Legitimacy, and Power. Nineteenth-Century Experiences, hrg. v. Kelly L. Grotke u. Markus J. Prutsch, Oxford: Oxford University Press, 2014, bes. 31-38. Darüber hinaus gab es natürlich vielfache Einflüsse aus der Verfassungstheorie, in diesem Fall den von Benjamin Constant, vgl. dazu António Manuel Hespanha, Guiando a mão invisivel. Direitos, Estado e lei no liberalismo monárquico português, Coimbra: Almedina, $2004,198$. Vgl. auch die Standarddarstellung von Paulo Bonavides / Paes de Andrade, História Constitucional do Brasil, 6. Aufl., Brasilia: OAB Editora, 2004, bes. 99-107. Generell zur brasilianischen Verfassungsgeschichte und Verfassungsgeschichtsschreibung Airton Cerqueira-Leite Seelaender, „Verfassung und Verfassungsrecht in Brasilien (1824-1988)“, in: Rechtsgeschichte, 16 (2010), 97103.

9 Documentos Constitucionales de Colombia y Panamá 1793-1853 / Constitutional Documents of Colombia and Panama 1793-1853, hrg. v. Bernd Marquardt, Berlin - New York: de Gruyter, 2010, 27-28.

10 Ebd., 29-60. 
Von diesen ersten Versuchen hebt sich die berühmte Constitución de Apatzingán von 1814 deutlich ab. ${ }^{11}$ Geradezu klassisch formulierte Art. 5 Volkssouveränität und repräsentatives System als Basis der Verfassung. Es folgten Gewaltentrennung (Art. 11 und 12), begrenzte Regierungsgewalt und Verantwortlichkeit der Regierenden (Art. 27) und Staatsbürgerrechte (Art. 24-40). Selbst wenn letztere nicht pauschal den Rang von allgemeinen Menschenrechten in Anspruch nehmen können, ${ }^{12}$ zeigt dies bei allen noch vorhandenen Beschränkungen doch, dass die Ideen des modernen Konstitutionalismus zu wirken begannen, so dass sie zumindest teilweise in die Gründungsdokumente der mexikanischen Föderation von 1824 einflossen. ${ }^{13}$

Mexiko war kein Einzelfall, wurde vielmehr sogar noch deutlich übertroffen von der Constitución de las Provincias unidas en Sud-America von 1819, die zumindest in Teilen die Kenntnis englischen wie nordamerikanischen Verfassungsdenkens erkennen ließ. Daher überrascht es nicht, dass die übernommenen Inhalte des modernen Konstitutionalismus sich überwiegend in der Declaración de derechos finden, selbst wenn diese erst am Ende der Verfassung folgt, wie es nicht allein der Bill of Rights der nordamerikanischen Bundesverfassung, sondern auch einer wachsenden Zahl von Einzelstaatsverfassungen der USA inzwischen entsprach. Hier finden sich Volkssouveränität (Art. CIV, CV), Gewaltentrennung und Verantwortlichkeit der Regierenden (Art. CVI), repräsentatives Regierungssystem (Art. CVIII) und Menschenrechte (Art. CIX-CXXIX). Die Unabhängigkeit der Justiz war zuvor schon festgestellt, indem die Richter des Obersten Gerichtshofes laut einer dann durchaus gängig werdenden Formulierung „exercerán el cargo por el tiempo de su buena comportacion“ - dies hatte dem Wortlaut des englischen Act of Settlement von 1701 entsprochen, dem Gründungsdokument der richterlichen Unabhängigkeit - und nicht gegen ihren Willen und den des Gerichtes versetzt werden konnten (Art. CII). Schließlich bedurften Verfassungsänderungen der

11 Generell zur Verfassung von Apatzingán Mariano Peset Reig, La Constitución de Apatzingán de 1814. Sentido y análisis de su texto, Puebla: Benemérita Universidad Autónoma de Puebla, 2014; La insurgencia mexicana y la Constitución de Apatzingán 1808-1824, hrg. v. Ana Carolina Ibarra et al., Mexico: Universidad Nacional Autónoma de México, 2014; Ernesto de la Torre Villar, La Constitución de Apatzingán y los creadores del Estado mexicano, 2. Aufl., Mexico: Universidad Nacional Autónoma de México, 2010, u.a.

12 Den Behauptungen von Barceló Rojas, es handele sich um „derechos humanos para todos“ (Daniel A. Barceló Rojas, „La Constitución de Apatzingán y su influencia en la primera generación de constituciones de la República Federal Mexicana“, in: La insurgencia mexicana y la Constitución de Apatzingán, hrg. v. Ibarra, 274) und von Torre Villar, es ginge hier um „los derechos del hombre, preexistentes a toda constitución, a toda ley y a toda sociedad“ (Torre Villar, La Constitución de Apatzingán, 57) kann in dieser Allgemeinheit nicht zugestimmt werden, da Kap. V ausdrücklich „De la igualdad, seguridad, propiedad y libertad de los ciudadanos“ handelt und lediglich fünf (Art. 31-35) der insgesamt 17 Artikel allgemeiner gefasst sind und keine Beschränkung auf „ciudadanos“ beinhalten. Staatsbürgerrechte sind ihrer Natur nach vom Staat für seine Bürger gesetzte Rechte, die mithin keine vorstaatliche Gültigkeit beanspruchen können. Bei Peset Reig, La Constitución de Apatzingán, 52-57, 108-113, findet sich diese Behauptung nicht. Im Gegenzug zitiert er zu den einzelnen Artikeln Konkordanzen in anderen Verfassungen, hier insbesondere den französischen Verfassungen von 1793 und 1795 und der Rechterklärung von Caracas von 1811.

13 Vgl. dazu auch Ivana Frasquet, „La soberanía en jaque. Liberalismo gaditano y república federal en México, 1820-1824“, in: El constitucionalismo mexicano. Influencias continentales y trasatlánticas, hrg. v. Patricia Galeana, México: Siglo XXI; Senado de la República, 2010, bes. 184192. 
indirekten Mitwirkung des Volkes (Art. CXXX-CXXXIV). Ob die ausdrückliche Auflistung der Befugnisse der Legislative wie der Exekutive in den Art. XXI-XXX und LXXIV-XCI einen hinreichenden Ersatz für die nicht deklarierte begrenzte Regierungsgewalt darstellt, mag dahingestellt bleiben. Hingegen kann die expressive Feststellung in Art. CVII „Ninguna autoridad del pais es superior á la ley“ kaum den fehlenden Verfassungsvorrang begründen, zumal es an einem wirklichen Verfassungsgericht fehlt. ${ }^{14}$

Dennoch stellte die Constitución de las Provincias unidas en Sud-America einen Meilenstein auf dem Weg zum modernen Konstitutionalismus dar, dem sich die Constitución politica de la República peruana von 1823 praktisch anschloss und in Teilen noch übertraf, wenngleich diese umgehend der Intervention Bolivars zum Opfer fiel und allein nach Ende seines Regimes 1827 nochmals für wenige Monate ins Leben gerufen wurde. Es finden sich hier nicht allein die genannten Bestimmungen der Constitución de Apatzingán, wobei sich deren Rechtsgarantien 1823 in Peru schon eher als Menschenrechte lesen ließen, bestimmte doch Art. 10, Peruaner seien „Todos los hombres libres nacido en el territorio del Perú“ sowie alle Naturalisierten, sei es durch Einbürgerung oder durch fünfjährige Anwesenheit. Sie alle fielen unter die Rechtegarantie der Verfassung laut Art. 193. Darüber hinaus wurde die Regierungsgewalt geradezu klassisch, und über die argentinischen Bestimmungen von 1819 hinausgehend, durch Art. 81 begrenzt, indem die „Limitaciones del Poder Ejecutivo“ sowohl vor exekutiven Amtsanmaßungen im Bereich der beiden anderen Gewalten schützten, als auch die Rechte und Freiheiten der Einzelnen bewahren sollten, wofür generell auch schon Art. 5 stand. Ferner fand sich hier der Einstieg in die Unabhängigkeit der Justiz ähnlich wie 1819 verankert, indem die Richter als „inamovibles“ erklärt wurden und auf Lebenszeit amtierten (Art. 97), und schließlich sollte die Verfassung allein durch einen "Congreso General“ mit aus allen Provinzen gewählten Deputierten verändert werden können (Art. 191, 192). ${ }^{15}$ Bis auf die Anrufung universeller Prinzipien ${ }^{16}$ und die Erklärung der Verfassung zum obersten Gesetz fanden sich alle zehn Prinzipien des modernen Konstitutionalismus in der peruanischen Verfassung von 1823, mehr als in jeder europäischen Verfassung zu diesem Zeitpunkt.

Doch statt diese Errungenschaften gebührend zu würdigen, hat ihr die Forschung einen „utopischen Liberalismus“ attestiert, von der sich die immer noch als liberal eingestufte Verfassung von 1828, die „Mutter“ aller nachfolgenden peruanischen Verfassungen bis einschließlich der von 1920,

14 Alle Zitate aus: Ma. Laura San Martino de Dromi, Documentos constitucionales argentinos, Buenos Aires: Ediciones Ciudad Argentina, 1994, 2318-2335.

15 Für den Verfassungstext und alle daraus zitierten Passagen, Darío Palacios Dextre u. Ruth Monge Guillergua, Las constituciones del Perú 1823-1993, Lima: Editora „Fecat“, 2004, 11 -34.

16 Aguilar Rivera sieht in dem bewussten Verzicht der Verfassung von Cádiz auf eine solide moderne naturrechtliche Begründung „una vulnerabilidad total en el edificio teórico y normativo del liberalismo hispánico. Esa debilidad sería luego transmitida a Hispanoamérica, tal y como ocurrió en México a mediados del siglo XIX, en donde esa fragilidad se hizo evidente en el transcurso de los debates entre liberales y conservadores“ (José Antonio Aguilar Rivera, "Memoria del código imposible: Cádiz y el experimento constitucional atlántico“, in: Cádiz a debate: actualidad, contexto y legado, hrg. v. Roberto Breña, Mexico: El Colegio de México, 2014, 122). 
deutlich unterschied. ${ }^{17}$ Tatsächlich war man, was die Prinzipien des modernen Konstitutionalismus betrifft, nunmehr in Peru deutlich hinter das Niveau der argentinischen Verfassung von 1819 und selbst der Constitución de Apatzingán zurückgefallen, während sich die Verfassung von Uruguay von $1830 \mathrm{im}$ wesentlichen dem Beispiel jenseits des La Plata anschloss - es fehlte das Verbot der richterlichen Versetzung wider Willen und die expressive Begrenzung der exekutiven Gewalt ${ }^{18}$-, und hinter der auch die Entwicklung in Chile im 19. Jahrhundert kaum zurück blieb. Manches klang dort mit der liberalen Verfassung von 1828 noch prononcierter, doch obwohl die konservativere Verfassung von 1833, die bis 1925 in Kraft war, wiederum die Rolle des Präsidenten hervorhob, fanden sich in ihr Volkssouveränität, repräsentatives Regierungssystem, Gewaltentrennung, Menschenrechte, Verantwortlichkeit der Regierenden, Ansätze zur Unabhängigkeit der Justiz und Abänderung der Verfassung unter indirekter Mitwirkung des Volkes. ${ }^{19}$

Das Beispiel Chile verdient insofern Beachtung, weil sich an ihm demonstrieren lässt, dass die für viele Teile Lateinamerikas im 19 und 20. Jahrhundert als konstitutiv geltende, schon aus den Unabhängigkeitsprozessen und der Diskussion um die Verfassung von Cadiz her rührende politische Trennung zwischen Liberalen und Konservativen die Auseinandersetzung um den modernen Konstitutionalismus nur begrenzt $\mathrm{zu}$ erklären vermag. ${ }^{20}$ Volkssouveränität, repräsentatives Regierungssystem, Gewaltentrennung und das eine oder andere weitere Prinzip waren für beide auf dem Papier akzeptabel und unstrittig. Doch selbst die am heftigsten umstrittenen Prinzipien wie die begrenzte Regierungsgewalt, die Unabhängigkeit der Justiz in allen ihren

17 Palacios Dextre u. Monge Guillergua, Las constituciones del Perú, 59. Ähnlich die Einschätzung der Verfassung von 1823 durch Giorgio Donati in: Le costituzioni dell'America latina, hrg. v. Eduardo Rozo Acuña und Giorgio Donati, 2 Bde., Rom: Senato della Reppublica, 20002004, II, 458-459. Schon der geachtete peruanische Intellektuelle, Jurist und nachmalige Außenminister Toribio Pacheco hatte 1854 das Wort geprägt, die Verfassung von 1823 „nació sólo para morir“, Toribio Pacheco, Cuestiones constitucionales (1854), 3. Aufl. m. e. Vorw. v. Domingo Garcia Belaunde, Lima: Grijley, 1996, 75.

18 Constitucion de la República Oriental del Uruguay, sancionada por la Asamblea general constituyente y legislative el 10 de Septiembre de 1829, Montevideo: Imprenta republicana, 1829 (für diese wie überhaupt die lateinamerikanischen Verfassungen der ersten Hälfte des 19. Jahrhunderts, vgl. meine Webseite http://www.modern-constitutions.de). Zwar hat diese bis 1917 in Kraft befindliche Verfassung in Art. 96 festgelegt, die Alta Corte de Justicia solle. Dass damit jedoch keine Verfassungsgerichtsbarkeit im heutigen Sinne gemeint war, stellte Art. 152 klar, gehörte es doch (ebd., 25, 34). Die Behauptung, die Verfassung beruhe auf, ist angesichts der inneren Widersprüchlichkeit zwischen den Dokumenten des modernen Konstitutionalismus und den napoleonischen Verfassungen wenig glaubwürdig, vgl. Ana Frega, in: El laboratorio constitucional iberoamericano, hrg. v. Annino und Ternavasio, 256.

$19 \mathrm{Zu}$ den chilenischen Verfassungen der ersten Hälfte des 19. Jahrhunderts, vgl. Documentos Constitucionales de Chile 1811-1833 / Constitutional Documents of Chile 1811-1833, hrg. v. Alan Bronfman, München: Saur, 2006. Vgl. Ana María Stuven, „De la autonomía a la república: El debate constitucional en Chile, 1808-1833“, in: El laboratorio constitucional iberoamericano, hrg. v. Annino und Ternavasio, 179-201; Bernd Marquardt, Staat, Verfassung und Demokratie in Hispano-Amerika seit 1810, Bd. I: Das liberale Jahrhundert, 1810-1916, Bogotá: Universidad Nacional de Colombia, Facultad de Derecho, 2008, 97-98.

20 Damit unterscheidet sich mein Ansatz von dem von Roberto Gargarella, The Legal Foundations of Inequality. Constitutionalism in the Americas, 1776-1860, New York: Cambridge University Press, 2010. In abgeschwächterer Form ebenso in Roberto Gargarella, Latin American Constitutionalism. The Engine Room of the Constitution, New York: Oxford University Press, 2013. 
Weiterungen oder der Rekurs auf universelle Prinzipien und damit die Einbindung in ein die Nation überschreitendes Werte- und Rechtssystem lassen sich nicht mit dem liberal/konservativen Erklärungsmuster fassen, stellten vielmehr Hürden dar, der eine traditionsbehaftete Fixierung auf die heroische Führungspersönlichkeit ebenso im Wege stand wie die nicht weniger belastete Verbindung zwischen Staat und Kirche, aus der sich auch die Liberalen in dieser Zeit kaum zu lösen vermochten, so dass schon auffällt, wenn die Constitución de las Provincias unidas en Sud-America in Art. I formuliert, dass zwar, wie überall, die katholische Religion Staatsreligion sei. Doch völlig abweichend von allen anderen dann fortfährt: „El gobierno le debe la mas eficaz y poderosa proteccion; y los habitantes del territorio todo respeto, qualesquiera que sean sus opiniones privadas". ${ }^{21}$ Erst die Verfassung von Nueva Granada von 1853 mit ihrem Bekenntnis zum Laizismus (Art. 5,5) sollte die enge Allianz zwischen Staat und katholischer Kirche aufbrechen. ${ }^{22}$

Die weitere Entwicklung erfolgte schrittweise. So enthielt die argentinische Verfassung von 1853, deren Beeinflussung durch die Verfassung der Vereinigten Staaten von Amerika an vielen Stellen erkennbar ist und die durch eine Reihe meist kleinerer Veränderungen zur Verfassung von 1860 wurde, die wiederum mit der Unterbrechung von 1949-1956 bis heute bei einer bislang letzten Amendierung von 1994 in Kraft ist, in ihrer Präambel einen ersten Anlauf zu dem, was man die Berufung auf universelle Prinzipien nennen könnte. Ein ausdrückliches Bekenntnis zur Volkssouveränität fehlte zwar, doch ließ die Verfassung in Art. 1 keinen Zweifel daran, dass die argentinische Nation zur Verfassungsgebung legitimiert sei. Neu war in jedem Fall der Art. 31, der die Verfassung zur „ley suprema de la Nacion“ erklärt, selbst wenn der Corte Suprema de Justitia die richterliche Überprüfung von Bundesgesetzen nicht eingeräumt wurde (vgl. Art. 100). Ein weiterer erster Schritt zur Begrenzung der Regierungsgewalt stellen Art. 28 und 29 dar, verboten sie doch einerseits die Einschränkung oder Abschaffung der garantierten Rechte durch entsprechende Gesetze sowie andererseits eine diktatoriale Machtusurpation („la suma del poder público“). Schließlich sollte die richterliche Unabhängigkeit durch Bestallung der Richter auf Lebenszeit und das Verbot der Minderung ihres Gehalts während ihrer Amtszeit erreicht werden, selbst wenn das Verbot der Versetzung wider Willen ebenso wie weitere Maßnahmen fehlten. ${ }^{23}$

Die mexikanische Verfassung von 1857, die mit der dreijährigen Unterbrechung des Kaiserreiches bis 1917 in Kraft blieb, wich von der argentinischen $\mathrm{ab}$, indem sie keine universellen Prinzipien, dafür jedoch ein klares Bekenntnis zur Volkssouveränität kannte. Wie diese erklärte es die

21 San Martin de Dromi, Documentos constitucionales argentinos, 2318-2319.

22 Überhaupt zeichnete ihr Rechtekatalog (Art. 5) und das allgemeine Männerwahlrecht (Art. 13) die Verfassung aus, die bei gewissen Modifikationen bis 1886 galt. Doch die bislang fehlenden Prinzipien - die Richter an der Suprema Corte de la Nacion sollten per Volkswahl auf vier Jahre gewählt werden - wurden auch hier nicht eingefügt, vgl. die Verfassung samt Annotationen in Documentos Constitucionales de Colombia y Panamá 1793-1853, hrg. v. Marquardt, 311-323.

23 Vgl. die Verfassung von 1853 und 1860 in San Martin de Dromi, Documentos constitucionales argentinos, 2527-2583 und unter http://www.verfassungen.net/ar/verf60-i.htm (Zugriff 27.7.2016). Das alles zusammen machte wohl das aus, was der Peronist Arturo Sampay als die „propios errores metafisicos“ dieser Verfassung abkanzelte, vgl. Arturo Enrique Sampay, La filosofía del iluminismo y la Constitución argentina de 1853, Buenos Aires: Depalma, $1944,44$. 
Verfassung zur „ley suprema de toda la Union“ (Art. 126). Konkrete Bestimmungen zur Begrenzung der Regierungsgewalt ähnlich denen in Argentinien fehlten, und auch die Unabhängigkeit der Richter am Obersten Gericht schien nicht wirklich gewährleistet. Sie wurden indirekt auf sechs Jahr gewählt (Art. 92), wobei zu einer etwaigen Wiederwahl nichts gesagt war, waren verantwortlich (Art. 103) und konnten bei ernsthaften Vorfällen von Kongress entlassen werden (Art. 95). Im Gegenzug fand sich hier erstmals ein Einstieg in eine Verfassungsgerichtsbarkeit (bes. Art. 101), ${ }^{24}$ die wiederum gemäß der Verfassung von 1880 der bolivianische Corte Suprema zukommen sollte, „cuya decisión dependa de la constitucionalidad o inconstitucionalidad de las leyes, decretos y cualquier género de resoluciones" (Art. 110,2). ${ }^{25}$

Nicht alle nachfolgenden Verfassungen anderer Staaten erreichten in der Folge dieses Niveau, blieben vielmehr bei dem einen oder anderen Prinzip des modernen Konstitutionalismus eine entsprechende Bestimmung oder korrespondierende Regelungen schuldig, so etwa die bereits indirekt erwähnte peruanische Verfassung von 1860, die kurzlebige liberale Verfassung von Ecuador von 1869, die in ihren Grundzügen bis 1944 wirksame Verfassung von Guatemala von $1879^{26}$ oder die Verfassung Kolumbiens von 1886, die bis 1991 in Kraft blieb, obgleich diese immerhin pauschal erklärte: „Todos los poderes públicos son limitados“ (Art. 57) und eine Verfassungsgerichtsbarkeit einfügte (Art. 151). ${ }^{27}$ Auch die brasilianische Verfassung von 1891 fiel, was die Prinzipien des modernen Konstitutionalismus betrifft, hinter das von Mexiko und Argentinien erreichte Niveau zurück. ${ }^{28}$

Besondere Erwähnung verdient hingegen die Verfassung von Paraguay von 1870, die bis 1940 in Kraft blieb und inhaltlich in vielen Punkten der argentinischen Verfassung nahe kam. Zwar blieb sie bei der richterlichen Unabhängigkeit (Amtsdauer vier Jahre mit Wiederwahl, Art. 112) zurück, erkannte aber ebenso die Verfassung als höchstes Gesetz an (Art. 16) und verfügte über bemerkenswerte Bestimmungen zur Begrenzung der Regierungsgewalt. So erklärte Art. 34, dass die Aufzählung von Rechten und Garantien nicht den Ausschluss der nicht genannten Rechte und Garantien bedeute, „pero que nacen del principio de la soberanía del pueblo y de la forma republicana democrática representativa“. Singulär war Art. 29: „Toda ley ó

24 Benutzt

nach http://www.cervantesvirtual.com/servlet/SirveObras/35716152323148053754491/thm0000.ht

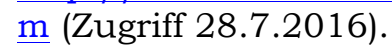

25 (http://comisiondeconstitucion2002-2003.awardspace.com/cpehistoria/cpe_1880.htm, Zugriff 29.7.2016). Ansonsten fiel die bolivianische Verfassung von 1880, die eine Modifikation der Verfassung von 1878 darstellte und bis 1938 wirksam blieb, generell in Bezug auf den modernen Konstitutionalismus hinter die mexikanische Verfassung zurück.

26 "longevidad común a los textos liberales definitivos": Jorge Mario García Laguardia, Breve historia constitucional de Guatemala, Guatemala: Ministerio de Cultura y Deportes, 2002, 66. Nicht allein die Verfassung von Chile von 1833 unterstreicht, dass Langlebigkeit im 19./20. Jahrhundert kein Alleinstellungsmerkmal liberaler Verfassungen war, ganz zu schweigen von den vielen kurzlebigen liberalen Verfassungen dieser Zeit.

27 Carlos Restrepo Piedrahita, Constituciones Politicas Nacionales de Colombia. Compilación, 2. Aufl., Bogotá: Universidad Externado de Colombia, 1995, 343-388. Vgl. Marquardt, Staat, Verfassung und Demokratie in Hispano-Amerika, I, 319-332.

28 Vgl. http://www.verfassungen.net/br/verf91-i.htm (Zugriff 28.7.2016). 
decreto que esté en oposición á lo que dispone esta Constitución, queda sin efecto y de ningún valor."29 Gleichwohl blieb die Frage, wie diese Bestimmung durchgesetzt werden konnte, zumal es an einem tatsächlichen Verfassungsgericht fehlte. Ähnlich herausragend war Art. 103: „Toda facultad ó atribución no delegada por esta Constitución al Poder Ejecutivo, carece in consecuencia de ella, correspondiendo al Congreso, como representación soberana del pueblo, dilucidar cualquiera duda llegara á haber en el equilibrio de los tres altos poderes del Estado." Schließlich fand sich der Art. 29 der argentinischen Verfassung hier in erweiterter Form als Art. 13 wieder, der alle außerordentlichen Befugnisse und die "suma del poder público" verbot ${ }^{30}$ und fortfuhr: "La dictadura es nula é inadmisible en la República del Paraguay“, eine Bestimmung, die hier wie in so manchem anderen lateinamerikanischen Land bedauerlicherweise nicht dem Test der Zeit standgehalten hat. ${ }^{31}$

Ziehen wir eine Zwischenbilanz, so wird man feststellen müssen, dass die Prinzipien des modernen Konstitutionalismus im Laufe des 19. Jahrhunderts dank eines verbreiteten Konsenses zwischen Liberalen und Konservativen in zunehmendem Maße in die Verfassungen Lateinamerikas Eingang fanden - eine Bilanz, die keinen Vergleich mit der Europas in dieser Zeit zu scheuen braucht. Zwar fiel das Gesamtergebnis von Land zu Land unterschiedlich aus, doch Volkssouveränität, repräsentative Regierung, Gewaltentrennung, Menschenrechte und Abänderung der Verfassung unter indirekter Mitwirkung des Volkes konnten praktisch überall als unstrittig gelten. Gleiches galt für die allseits gerne reklamierte Verantwortlichkeit der Regierenden. Die Notwendigkeit, die Verfassung auf universellen Prinzipien zu begründen, hatte sich hingegen bislang kaum einem lateinamerikanischen Verfassungsgeber erschlossen. Dieses Prinzip bildet mit den drei verbliebenen den eigentlichen Kern für die Eigentümlichkeiten der lateinamerikanischen Verfassungsentwicklungen, den viele in dem betonten Präsidentialismus Lateinamerikas sehen und der, bezogen auf den modernen Konstitutionalismus, die überzeugende Umsetzung des Prinzips der begrenzten Regierungsgewalt so lückenhaft macht und selbst dort, wo es in der Verfassung deklariert wurde, nicht zu überzeugenden Mechanismen zwecks seiner tatsächlichen Durchsetzung führte. Eine zweiter, damit eng verbundener Bereich sind die traditionelle Rolle und das Verständnis von Recht. Dieses äußert sich nicht allein in dem, von den wenigen genannten Ausnahmen abgesehenen durchgängigen Fehlen der Erklärung der Verfassung zum obersten Gesetz - der

29 Sehr ähnlich formulierte die Verfassung von Costa Rica von 1871 in Art. 17: „Las disposiciones del Poder Legislativo ó del Ejecutivo que fueren contrarias á la Constitución son nulas y de ningún valor, cualquiera que sea la forma en que se emitan" (Constitución politica de la República de Costa-Rica 7 de Diciembre de 1871. Reformada en 1882, 1886 y 1889, San José: o.N., $1889,16)$. Auch in diesem Fall blieb offen, wie diese Bestimmung durchgesetzt werden sollte. Die liberale Verfassung, die für die Geschichte Costa Ricas prägend wurde, erreichte in Bezug auf den modernen Konstitutionalismus nicht vollends das Niveau der von Paraguay und blieb mit der Unterbrechung von 1917-1924 bis 1949 in Kraft.

30 Auch die bolivianische Verfassung von 1880 kannte diese Bestimmung (Art. 30), wobei ungeklärt erscheint, wie diese mit den ungewöhnlich ausführlichen Regelungen über den Belagerungszustand zu vereinbaren waren, vgl. Art. 26-29 (http://comisiondeconstitucion20022003.awardspace.com/cpehistoria/cpe_1880.htm, Zugriff 29.7.2016), die sich bis heute in vielen lateinamerikanischen Verfassungen finden lassen.

31 Die Verfassungszitate wurden entnommen: Fernando Viera, Colleción Legislativa de la República del Paraguay, Asunción: H. Kraus, 1896, 9-31. 
in der Verfassungswirklichkeit eine mitunter verbreitete Geringschätzung der Verfassung entsprach -, sondern, unmittelbar damit verbunden, in dem gesamten Bereich der richterlichen Gewalt. Zwar hat so gut wie keine Verfassung ein Problem damit, die Justiz als dritte Gewalt verbal einzusetzen. Dennoch blieb dies, ungeachtet umfangreicher Befugniskataloge, in aller Regel verfassungsrechtlich eine Leerstelle. Nirgendwo wird als Prinzip die richterliche Unabhängigkeit verbal proklamiert, und es erscheint fast beliebig, ob Richter auf Lebenszeit eingesetzt oder auf wenige Jahre, häufig mit dem Recht auf Wiederwahl, gewählt wurden. Die Verfassungsimplikationen wurden, so muss man wohl daraus folgern, nicht gesehen, selbst wenn es in einigen Fällen ergänzende Bestimmungen gegeben haben mag. Die Rolle der Richter wie der Gerichte als Wahrer des Rechts und damit auch des obersten Rechts, nämlich der Verfassung, erscheint auch am Ende des 19. Jahrhunderts in der Regel noch so weit weg von dem auf die Exekutive und ihre Gewalt fixierten Blick, dass dieser in der Praxis - und hier oftmals verbunden mit Korruption und Klientelismus wie Nepotismus - nur zu leicht zur Gefahr für die Verfassung selbst wurde.

Richten wir daher abschließend den Blick konkret auf diese kritischen Prinzipien im 20. Jahrhundert und betrachten zunächst Mexiko mit seiner Verfassung von 1917 als eine der ältesten nationalen Verfassungen und der aktuell in Lateinamerika in Kraft befindlichen Verfassungen einschließlich ihrer seither erfolgten Amendierungen. Auch weiterhin fehlt ein expressives Bekenntnis zu universellen Prinzipien, für das die Präambel der klassische Ort wäre. Ob dafür die wiederholten Berufungen auf die internationalen Verpflichtungen Mexikos sowie die herausgehobene Betonung der Menschenrechte als angemessener Ersatz gelten kann, mag dahin gestellt sein. Ebenso vermisst man die ausdrückliche Erklärung der Verfassung zum obersten Gesetz - vergleichbar etwa dem Art. 39 der Verfassung von Querétaro von 2008, in der von der „Norma Fundamental del Estado" die Rede ist ${ }^{32}$-, doch kennt die Verfassung seit 1917 den Art. 136 „De la inviolabilidad de la Constitución“. Die zwar auch weiterhin nicht ausdrücklich erklärte Unabhängigkeit der Justiz wird durch eine gegenüber früher deutlich gestärkte richterliche Gewalt mit einem kraftvollen Verfassungsgericht zum Ausdruck gebracht, dessen Richter auf 15 Jahre bestellt werden und darauf nicht wieder ernannt werden können (vgl. dazu insgesamt Art. 94-107). Auch die Begrenzung der exekutiven Gewalt wird nicht ausdrücklich thematisiert, jedoch erscheinen die exekutiven Befugnisse nicht nur strikter gefasst. Auch der neu formulierte Titel IV (Art. 108-114) über die Verantwortlichkeit der Staatsdiener und den Tatbestand der Korruption kann als gravierender Versuch gewertet werden, Barrieren zu errichten. ${ }^{33}$

Ungeachtet ihres ganz anderen Charakters wies die Verfassung von Costa Rica von 1949 die gleichen vier Lücken auf. Doch auch hier sorgten ein Verfassungsgericht (Art. 10) ${ }^{34}$ und eine weit gefasste Verantwortlichkeit (Art. 148-

32 Constitución política del Estado de Querétaro (Fe de erratas P. O. No. 21, 11-IV-08).

33 Vgl. die aktuell gültige Verfassung unter Einschluss der bislang letzten Reform vom 27.1.2016 unter http://www.diputados.gob.mx/LeyesBiblio/htm/1.htm (Zugriff 30.7.2016).

$34 \mathrm{Zu}$ der maßstabsetzenden Rolle Costa Ricas in der Verfassungsgerichtsbarkeit seit der Verfassungsänderung von 1989, vgl. José Fabián Ruiz Valerio, ¿Democracia o Constitución? El debate actual sobre el Estado de derecho, Mexico: Fontamara, 2009, bes. 197-202. 
151), wengütigegütigegütigegütigegütigen auch ohne besondere Thematisierung der Korruption, für Barrieren, ergänzt durch das ausdrückliche Verbot der Suspendierung von Rechten und individuellen Garantien (Art. 121,7). Hingegen wurden die obersten Richter auf acht Jahre, bei der Möglichkeit der Wiederwahl bestellt (Art. 158), konnten aber nur in außergewöhnlichen Fällen suspendiert werden (Art. 165). ${ }^{35}$

In der zweiten Hälfte des 20. Jahrhunderts, und insbesondere seit den 1980er Jahren, wandelte sich dieses traditionelle Bild der lateinamerikanischen Verfassungen, ${ }^{36}$ so dass heute die Verfassung von Argentinien von 1994, in der offiziellen Lesart lediglich die bislang letzte Modifikation der Verfassung von 1853, eher als Relikt einer vergangenen Zeit erscheint, was schon das Bekenntnis zur katholischen Kirche in Art. 2 zum Ausdruck bringt, das unverändert dem Wortlaut des Art. 2 der Verfassung von 1853 entspricht, ${ }^{37}$ während alle übrigen lateinamerikanischen Verfassungen der letzten vier Jahrzehnte die Trennung von Staat und Kirche vollzogen haben. ${ }^{38}$

Universelle Prinzipien, ${ }^{39}$ die Erklärung der Verfassung zum obersten Gesetz, ${ }^{40}$ begrenzte Regierung ${ }^{41}$ und die richterliche Unabhängigkeit ${ }^{42}$ sind seit den 1980er Jahren in die lateinamerikanischen Verfassungen eingezogen und wiederholt mit Nachdruck betont. Man könnte also zu dem Schluss kommen, dass schließlich im Kontext des Siegeszuges der Demokratie die Prinzipien des modernen Konstitutionalismus in Lateinamerika angekommen sind bei allen Unterschieden, die es zwischen den Verfassungen der einzelnen Staaten unverändert gibt. Ein derartiges Fazit würde jedoch die Eigentümlichkeiten des lateinamerikanischen Konstitutionalismus kaum hinreichend erklären.

35 Vgl. die aktuell gültige Fassung unter

http://www.pgrweb.go.cr/scij/Busqueda/Normativa/Normas/nrm_texto_completo.aspx?param1= NRTC\&nValor $1=18$ nValor2=8718nValor3=95479\&strTipM=TC (Zugriff 30.7.2016).

36 Auf die weltweiten Gründe für die Konsolidierungsphase der Demokratie seit den 1970er Jahren, vgl. José Gamas Torruco, „Globalización, constitucionalismo y transiciones democráticas“, in: El constitucionalismo mexicano, hrg. v. Galeana, bes. 271-277.

37 Vgl. http://servicios.infoleg.gob.ar/infolegInternet/anexos/0-4999/804/norma.htm (Zugriff 2.8.2016); San Martin de Dromi, Documentos constitucionales argentinos, 2528.

38 Generell zur Einordnung der Verfassung von 1994, vgl. Abelardo Levaggi, „Three Matters Concerning Argentine Constitutional History“, In: Rechtsgeschichte, 16 (2010), 82-84.

39 Sie lassen sich etwa aus der Verfassung von Honduras von 1982, Art. 15, und der Verfassung von Venezuela von 1999, Präambel, herauslesen.

40 Vgl. Verfassung von Honduras von 1982, Art. 320. Verfassung von Guatemala von 1985, Art. 175, Verfassung von Nicaragua von 1986, Art. 182, Verfassung von Kolumbien von 1991, Art. 4, Verfassung von Paraguay von 1992, Art. 137, Verfassung von Perú von 1993, Art. 51, Verfassung von Argentinien von 1994, Art. 31, Verfassung von Venezuela von 1999, Art. 334, Verfassung von Ecuador von 2008, Art. 424, Verfassung von Bolivien von 2009, Art. 410,II.

41 Vgl. Verfassung von Guatemala von 1985, Art. 152, Verfassung von Brasilien von 1988, Art. 85-86, Verfassung von Paraguay von 1992, Art. 193-194, 225, auch Verfassung von Ecuador von 2008, Art. 129-131.

42 Vgl. Verfassung von Honduras von 1982, Art. 303, Verfassung von E1 Salvador von 1983, Art. 172, Verfassung von Brasilien von 1988, Art. 95, Verfassung von Kolumbien von 1991, Art. 228, 233, Verfassung von Paraguay von 1992, Art. 248, Verfassung von Perú von 1993, Art. 146, Verfassung von Venezuela von 1999, Art. 254, 256, Verfassung von Ecuador von 2008, Art. 168, Verfassung von Bolivien von 2009, Art. 178. 
Dieser moderne Konstitutionalismus, der sich schon in den Anfängen der Verfassungsgebung der um ihre Unabhängigkeit kämpfenden neuen Staaten dokumentierte, musste von Anbeginn seinen Platz in einem politisch-kulturellen Umfeld finden, das nicht nur völlig anders geprägt war als das angloamerikanische Nordamerika im ausgehenden 18. Jahrhundert, sondern für das sich auch im 19. und 20. Jahrhundert die Vereinigten Staaten von Amerika lediglich in den seltensten Fällen als politisches Modell anbot. Doch es gab nicht allein diese politisch-mentalen Barrieren gegenüber einer Macht, die nur zu oft als arrogant und imperialistisch wahrgenommen wurde. Die englischen Siedler im Nordamerika des 17. und 18. Jahrhunderts hatten ein anderes kulturelles Gepäck mitgebracht als die spanischen und portugiesischen Eroberer Lateinamerikas und ihre Nachfahren mit ihrem mitunter lange nachwirkenden scholastisch-katholischen und antiindividualistischen Weltbild. ${ }^{43}$ Der englische Begriff der rule of law war kein vergleichbares Element der iberischen Staatsphilosophie gewesen. ${ }^{44}$ Selbst in dem heute in den Verfassungen durchaus anzutreffenden Bekenntnis zum estado de derecho ${ }^{45} \mathrm{klingt}$ dies, nicht allein wegen der vielfach angefügten sprachlichen Erweiterungen, nicht selten anders und kaum nach Harringtons „empire of laws and not of men“.46 Der lateinamerikanische estado de derecho, so hat man den Eindruck, präsentiert sich gerne als ein empire of men - selbst wo in heutigen Verfassungen nicht von Heroen und Märtyrern die Rede ist -, der mitunter seine Personalisierung in einem defensor del pueblo findet. ${ }^{47}$

Angesichts des lange Lateinamerika prägenden Präsidentialismus und caudillismo erscheinen diese Konstruktionen zur Einhegung der Verfassung zwar nachvollziehbar, schien doch die einfache, in sich ausbalancierte Verfassung des modernen Konstitutionalismus, die, wie die Geschichte hinreichend unter Beweis gestellt hat, lediglich einen mit Inhalt zu füllenden strukturellen Rahmen setzt

43 Vgl. dazu die Überlegungen von Eric Eduardo Palma González, „Die moralische Frage bei der Bildung des Verfassungsstaats. Chile im 19. Jh.: ein katholischer, liberaler Staat aus praktizierenden Regierenden und Bürgern“, in: Rechtsgeschichte, 16 (2010), 108-110. Sehr viel begrenzter José Maria Portillo Valdés, „Early Constitutionalism and the Limits of Liberalism in the Spanish World“. in: Constitutional Cultures: On the Concept and Representation of Constitutions in the Atlantic World, hrg. v. Silke Hensel et al., Newcastle upon Tyne: Cambridge Scholars Publishing, 2012, 43-66.

44 Die Verneinung dieses Unterschieds durch die amerikanische Politologin Karen Orren erscheint mir zu formalistisch gedacht, vgl. Karen Orren, „Constitutional Development in the United States and Argentina“, in: Globality and Multiple Modernities. Comparative North American and Latin American Perspectives, hrg. v. Luis Roniger und Carlos H. Waisman, Brighton Portland, Ore.: Sussex Academic Press, 2002, 117-132.

45 So unter andern Verfassung von Perú von 1993, Art. 3. Vgl. dazu u.a. Ruiz Valerio, ¿Democracia o Constitución?.

46 James Harrington, The Commonwealth of Oceana [1656], in: The Political Works of James Harrington, hrg. v. J. G. A. Pocock, Cambridge: Cambridge University Press, 1977, 171.

47 Vgl. Verfassung von Paraguay von 1992, Art. 276-280, Verfassung von Perú von 1993, Art. 161-162, Verfassung von Argentinien von 1994, Art. 86, Verfassung von Venezuela von 1999 , Art. 280-283, Verfassung von Ecuador von 2008, Art. 214-216, Verfassung von Bolivien von 2009, Art. 218-224. Vgl. auch die rechtstheoretische Untersuchung zur politischen und rechtlichen Kontrolle im demokratischen Verfassungsstaat von Manuel Aragón, Constitución, democracia y control, Mexico: Universidad Nacional Autónoma de México, 2002, bes. 81-213. 
und damit offen für die Probleme der Zukunft ist, ${ }^{48}$ angesichts der spezifischen lateinamerikanischen Gegebenheiten ohne zusätzliche Hilfskonstruktionen nicht erreichbar. Das hat mitunter zu einer letztlich kontraproduktiven Aufblähung einzelner neuerer Verfassungen geführt, deren endlose Rechtekataloge und minutiöse Einzelregelungen zu Widersprüchlichkeiten und einer inhärenten Durchsetzungsschwäche führen, die rasche Verfassungsänderungen, wenn nicht gar Verfassungsmissachtungen geradezu provozieren werden. Erst die Zukunft wird zeigen, ob begrenzte Regierungsgewalt und unabhängige Justiz mit einem kraftvollen, die Rechte der Bürger aktiv verteidigenden Verfassungsgericht ${ }^{49}$ zur Sicherung von Freiheit und Rechtsstaatlichkeit führen und dann möglicherweise gar die heute noch erforderlichen Hilfskonstruktionen überflüssig machen werden. Auch außerhalb Lateinamerikas war dies oftmals ein jahrzehntelanger Prozess, und nicht überall erscheint das Ziel bereits erreicht. Gerade die europäische Geschichte hat aber auch gelehrt, dass angesichts der Schwierigkeiten auf diesem Weg das Aufgeben einer republikanischdemokratischen Verfassungsordnung einen gefährlichen Irrweg darstellt.50 Versuche, stattdessen radikal neue Wege zu gehen, wie etwa in Bolivien, erscheinen dagegen ungeachtet der zwingenden Notwendigkeit, benachteiligte ethnische Gruppen unter Wahrung ihrer kulturellen Identitäten in den Staat zu integrieren, ideologisch überbefrachtet und in sich $\mathrm{zu}$ widersprüchlich, um sich als zukunftsfähiges Modell zu empfehlen. ${ }^{51}$

Fecha de envío / Sumission date: $7 / 12 / 2019$

Fecha de aceptación / Acceptance date: 4/01/2020

$48 \mathrm{Zu}$ der verbreiteten Diskussion, ob das 21. Jahrhundert einen neuen Konstitutionalismus braucht, vgl. u.a. Manuel Sánchez de Diego y Fernández de la Riva, „Nuevos horizontes para el constitucionalismo del siglo XXI“, in: El constitucionalismo mexicano, hrg. v. Galeana, 371-382; Horst Dippel, „Braucht Lateinamerika einen neuen Konstitutionalismus?“, in: Rechtsgeschichte, 16 (2010), 32-34.

49 Zum richterlichen Prüfungsrecht heute, vgl. Verfassung von Honduras von 1982, Art. 184186, Verfassung von El Salvador von 1983, Art. 174, 183, Verfassung von Guatemala von 1985, Art. 272, Verfassung von Nicaragua von 1986, Art. 164, 187-190, Verfassung von Brasilien von 1988, Art. 97, 102, Verfassung von Kolumbien von 1991, Art. 241, Verfassung von Paraguay von 1992, Art. 132, 260, Verfassung von Perú von 1993, Art. 201-204, Verfassung von Venezuela von 1999, Art. 266, 334. Zu dieser neuen aktiven Rolle von Verfassungsgerichten in Lateinamerika heute generell, vgl. Joaquín Garcia-Huidobro, „Der schwierige hispanoamerikanische Konstitutionalismus“, in: Rechtsgeschichte, 16 (2010), 40-46.

50 So etwa Juan Fernando Segovia, „Die Entkräftung des hispanoamerikanischen Konstitutionalismus“, in: Rechtsgeschichte, 16 (2010), 35-39, der darin ein Lateinamerika von Europa aufgezwungenes Prokrustesbett sieht.

51 Vgl. dazu u.a. Claudia Josi, Overcoming the Crisis: Diversity and Human Rights in the New Bolivian Constitution, Bern: Swisspeace, 2014. 